Bull. Korean Math. Soc. 52 (2015), No. 6, pp. 2071-2093

http://dx.doi.org/10.4134/BKMS.2015.52.6.2071

\title{
SIMILAR AND SELF-SIMILAR CURVES IN MINKOWSKI $n$-SPACE
}

\author{
Mustafa ÖZdemir and HaKan Simsek
}

\begin{abstract}
In this paper, we investigate the similarity transformations in the Minkowski $n$-space. We study the geometric invariants of nonnull curves under the similarity transformations. Besides, we extend the fundamental theorem for a non-null curve according to a similarity motion of $\mathbb{E}_{1}^{n}$. We determine the parametrizations of non-null self-similar curves in $\mathbb{E}_{1}^{n}$.
\end{abstract}

\section{Introduction}

A similarity transformation (or similitude) of Euclidean space, which consists of a rotation, a translation and an isotropic scaling, is an automorphism preserving the angles and ratios between lengths. The geometric properties unchanged by similarity transformations is called the similarity geometry. The whole Euclidean geometry can be considered as a glass of similarity geometry. The similarity transformations are studying in most area of the pure and applied mathematics.

Curve matching is an important research area in the computer vision and pattern recognition, which can help us determine what category the given test curve belongs to. Also, the recognition and pose determination of $3 \mathrm{D}$ objects can be represented by space curves are important for industry automation, robotics, navigation and medical applications. S. Li [22] showed an invariant representation based on so-called similarity-invariant coordinate system (SICS) for matching 3D space curves under the group of similarity transformations. He also [21] presented a system for matching and pose estimation of 3D space curves under the similarity transformation. Brook et al. [7] discussed various problems of image processing and analysis by using the similarity transformation. Sahbi [28] investigated a method for shape description based on kernel principal component analysis (KPCA) in the similarity invariance of KPCA.

Received November 11, 2014; Revised February 9, 2015.

2010 Mathematics Subject Classification. 53A35, 53A55, 53B30.

Key words and phrases. Lorentzian similarity geometry, similarity transformation, similarity invariants, similar curves, self-similar curves. 
There are many applications of the similarity transformation in the computer vision and pattern recognition (see also $[1,30]$ ).

The idea of self-similarity is one of the most basic and fruitful ideas in mathematics. A self-similar object is exactly similar to a part of itself, which in turn remains similar to a smaller part of itself, and so on. In the last few decades it established itself as the central notion in areas such as fractal geometry, dynamical systems, computer networks and statistical physics. Recently, the self-similarity started playing a role in algebra as well, first of all in group theory $([17,25])$. Mandelbrot presented the first description of self-similar sets, namely sets that may be expressed as unions of rescaled copies of themselves. He called these sets fractals, which are systems that present such self-similar behavior and the examples in nature are many. The Cantor set, the von Koch snowflake curve and the Sierpinski gasket are some of the most famous examples of such sets. Hutchinson and, shortly thereafter, Barnsley and Demko showed how systems of contractive maps with associated probabilities, referred to as Iterated Function Systems (IFS), can be used to construct fractal, self-similar sets and measures supported on such sets (see [3, 4, 14, 19, 23]).

When the n-dimensional Euclidean space $\mathbb{E}^{n}$ is endowed with the Lorentzian inner product, we obtain the Lorentzian similarity geometry. The Lorentzian flat geometry is inside the Lorentzian similarity geometry. Aristide [2] investigated the closed Lorentzian similarity manifolds. Kamishima [20] studied the properties of compact Lorentzian similarity manifolds using developing maps and holonomy representations. The geometric invariants of curves in the Lorentzian similarity geometry have not been considered so far. The theme of similarity and self-similarity will be interesting in the Lorentzian-Minkowski space.

Many integrable equations, like Korteweg-de Vries (mKdV), sine-Gordon and nonlinear Schrödinger (NLS) equations, in soliton theory have been shown to be related to motions of inextensible curves in the Euclidean space. By using the similarity invariants of curves under the similarity motion, K.-S. Chou and C. $\mathrm{Qu}[9]$ showed that the motions of curves in two-, three- and $n$-dimensional $(n>3)$ similarity geometries correspond to the Burgers hierarchy, BurgersmKdV hierarchy and a multi-component generalization of these hierarchies in $\mathbb{E}^{n}$. Moreover, to study the motion of curves in the Minkowski space also attracted researchers' interest. Gürses [18] studied the motion of curves on two-dimensional surface in Minkowski 3-space. Q. Ding and J. Inoguchi [10] showed that binormal motions of curves in Minkowski 3-space are equivalent to some integrable equations. In the 4-dimensional Minkowski space Nakayama [24] presented a formulation on the motion of curves in hyperboloids, which includes many equations integrable by means of the 1+1-dimensional AKNS inverse scattering scheme. Therefore, the current paper can contribute to study the motion of curves with similarity invariants in $\mathbb{E}_{1}^{n}$.

Berger [6] represented the broad content of similarity transformations in the arbitrary dimensional Euclidean spaces. Encheva and Georgiev [12, 13] studied 
the differential geometric invariants of curves according to a similarity in the finite dimensional Euclidean spaces. In the current paper, Lorentzian version of similarity transformations will be entitled by pseudo-similarity transformation defined by (1) in Section 2. The main idea of this paper is to extend the fundamental theorem for a non-null curve with respect to p-similarity motion and determine non-null self-similar curves in the Minkowski $n$-space $\mathbb{E}_{1}^{n}$.

The scope of paper is as follows. First, we prove that the p-similarity transformations preserve the causal characters and angles. We introduce differential geometric invariants of a non-lightlike Frenet curve which are called p-shape curvatures according to the group of p-similarity transformations in $\mathbb{E}_{1}^{n}$. We give the uniqueness theorem which states that two non-null curves having same the p-shape curvatures are equivalent according to a p-similarity. Furthermore, we obtain the existence theorem that is a process for constructing a non-null curve by its p-shape curvatures under some initial conditions. Lastly, we study an exact description of non-null self-similar curves in $\mathbb{E}_{1}^{n}$. Especially, we examine the low-dimensional cases $n=2,3,4$ in more details.

\section{Fundamental group of the Lorentzian similarity geometry}

Firstly let us give some basic notions of the Lorentzian geometry. Let $\mathbf{x}=$ $\left(x_{1}, \ldots, x_{n}\right)^{T}$ and $\mathbf{y}=\left(y_{1}, \ldots, y_{n}\right)^{T}$ be two arbitrary vectors in the Minkowski space $\mathbb{E}_{1}^{n}$. The Lorentzian inner product of $\mathbf{x}$ and $\mathbf{y}$ can be stated as $\mathbf{x} \cdot \mathbf{y}=$ $\mathbf{x}^{T} I^{*} \mathbf{y}$ where $I^{*}=\operatorname{diag}(-1,1,1, \ldots, 1)$. Then, the norm of the vector $\mathbf{x}$ is represented by $\|\mathbf{x}\|=\sqrt{|\mathbf{x} \cdot \mathbf{x}|},[16,26]$.

Theorem 1. Let $\mathbf{x}$ and $\mathbf{y}$ be vectors in the Minkowski $n$-space $\mathbb{E}_{1}^{n}$.

(i) If $\mathbf{x}$ and $\mathbf{y}$ are timelike vectors which are in the same timecone of $\mathbb{E}_{1}^{n}$, then there is a unique number $\theta \geq 0$, called the hyperbolic angle between $\mathbf{x}$ and $\mathbf{y}$ such that $\mathbf{x} \cdot \mathbf{y}=-\|\mathbf{x}\|\|\mathbf{y}\| \cosh \theta$.

(ii) If $\mathbf{x}$ and $\mathbf{y}$ are spacelike vectors satisfying the inequality $|\mathbf{x} \cdot \mathbf{y}|<\|\mathbf{x}\|\|\mathbf{y}\|$, then $\mathbf{x} \cdot \mathbf{y}=\|\mathbf{x}\|\|\mathbf{y}\| \cos \theta$ where $\theta$ is the angle between $\mathbf{x}$ and $\mathbf{y}$.

(iii) If $\mathbf{x}$ and $\mathbf{y}$ are spacelike vectors satisfying the inequality $|\mathbf{x} \cdot \mathbf{y}|>\|\mathbf{x}\|\|\mathbf{y}\|$, then $\mathbf{x} \cdot \mathbf{y}=\|\mathbf{x}\|\|\mathbf{y}\| \cosh \theta$ where $\theta$ is the hyperbolic angle between $\mathbf{x}$ and $\mathbf{y}$.

Now, we define similarity transformation in $\mathbb{E}_{1}^{n}$. A pseudo-similarity ( $p$ similarity) of Minkowski n-space $\mathbb{E}_{1}^{n}$ is a composition of a dilatation (homothety) and a Lorentzian motion. Any p-similarity $f: \mathbb{E}_{1}^{n} \rightarrow \mathbb{E}_{1}^{n}$ is determined by

$$
f(x)=\mu \mathbf{A} x+\mathbf{b},
$$

where $\mu$ is a real constant, $\mathbf{A}$ is a fixed pseudo-orthogonal $n \times n$ matrix with $\operatorname{det}(\mathbf{A})=1$ and $\mathbf{b}=\left(b_{1}, \ldots, b_{n}\right)^{T} \in \mathbb{R}_{1}^{n}$ is a translation vector. When $n$ is odd and $\mu$ is a positive real constant or $n$ is even and $\mu$ is a non-zero real constant, $f$ is an orientation-preserving similarity transformation. When $n$ is odd and $\mu$ is a negative real constant, $f$ is an orientation-reversing p-similarity transformation. 
Since $f$ is a affine transformation, we get $\vec{f}(\mathbf{u})=\mu \mathbf{A u}$ and $\|\vec{f}(\mathbf{u})\|=|\mu|\|\mathbf{u}\|$ for any $\mathbf{u} \in \mathbb{R}_{1}^{n}$ where $\vec{f}(\overrightarrow{x y})=\overrightarrow{f(x) f(y)}$ (see $[6]$ ). The constant $|\mu|$ is called p-similarity ratio of the transformation $f$. The p-similarity transformations are a group under the composition of maps and we denote by $\operatorname{Sim}\left(\mathbb{E}_{1}^{n}\right)$. This group is a fundamental group of the Lorentzian similarity geometry. Also, the group of orientation-preserving (reversing) p-similarities are denoted by $\operatorname{Sim}^{+}\left(\mathbb{E}_{1}^{n}\right)$ $\left(\operatorname{Sim}^{-}\left(\mathbb{E}_{1}^{n}\right)\right.$, resp. $)$.

Theorem 2. The p-similarity transformations preserve the causal characters and angles.

Proof. Let $f$ be a p-similarity. Then, since we can write the equation

$$
\vec{f}(\mathbf{u}) \cdot \vec{f}(\mathbf{u})=\mu^{2}(\mathbf{A u} \cdot \mathbf{A} \mathbf{u})=\mu^{2}(\mathbf{u} \cdot \mathbf{u}),
$$

$f$ preserves the causal character in $\mathbb{E}_{1}^{n}$.

Let $\mathbf{u}$ and $\mathbf{v}$ are timelike vectors which are in the same timecone of $\mathbb{E}_{1}^{n}$. We consider $\theta$ and $\gamma$ as the angles between $\mathbf{u}, \mathbf{v}$ and $\vec{f}(\mathbf{u}), \vec{f}(\mathbf{v})$, respectively. Since $\vec{f}(\mathbf{u})$ and $\vec{f}(\mathbf{v})$ have same causal characters with $\mathbf{u}$ and $\mathbf{v}$, we can find the following equation from Theorem 1;

$$
\begin{aligned}
\vec{f}(\mathbf{u}) \cdot \vec{f}(\mathbf{v}) & =-\|\vec{f}(\mathbf{u})\|\|\vec{f}(\mathbf{v})\| \cosh \gamma, \\
\mu^{2}(\mathbf{u} \cdot \mathbf{v}) & =-\mu^{2}\|\mathbf{u}\|\|\mathbf{v}\| \cosh \gamma, \\
-\|\mathbf{u}\|\|\mathbf{v}\| \cosh \theta, & =-\|\mathbf{u}\|\|\mathbf{v}\| \cosh \gamma, \\
\cosh \theta & =\cosh \gamma .
\end{aligned}
$$

From here, we have $\theta=\gamma$. If $\mathbf{u}$ and $\mathbf{v}$ are spacelike vectors satisfying the inequality $|\mathbf{u} \cdot \mathbf{v}|<\|\mathbf{u}\|\|\mathbf{v}\|$, then

$$
\|\vec{f}(\mathbf{u})\|\|\vec{f}(\mathbf{v})\|=\mu^{2}\|\mathbf{u}\|\|\mathbf{v}\|>\mu^{2}|\mathbf{u} \cdot \mathbf{v}|=|\vec{f}(\mathbf{u}) \cdot \vec{f}(\mathbf{v})| .
$$

Therefore, it can be said from Theorem 1 that we have $\theta=\gamma$ similar to (3) .

It can also be found that $\theta$ is equal to $\gamma$ in case of condition (iii) in Theorem 1 . As a consequence, every p-similarity transformation preserves the angle between any two vectors.

\section{Geometric invariants of non-null curves in Lorentzian similarity geometry}

Let $\alpha: t \in J \rightarrow \alpha(t) \in \mathbb{E}_{1}^{n}$ be a non-null curve of class $C^{n}$ and $\left\{\mathbf{e}_{1}, \ldots, \mathbf{e}_{n}\right\}$ be a Frenet moving n-frame of $\alpha$ where $J \subset \mathbb{R}$ is an open interval. We denote image of $\alpha$ under $f \in \operatorname{Sim}\left(\mathbb{E}_{1}^{n}\right)$ by $\alpha^{*}$, i.e., $\alpha^{*}=f \circ \alpha$. Then, $\alpha^{*}$ can be stated as

$$
\alpha^{*}(t)=\mu \mathbf{A} \alpha(t)+b, \quad t \in I
$$


The arc length functions of $\alpha$ and $\alpha^{*}$ starting at $t_{0} \in J$ are

$$
s(t)=\int_{t_{0}}^{t}\left\|\frac{d \alpha(u)}{d u}\right\| d u, \quad s^{*}(t)=\int_{t_{0}}^{t}\left\|\frac{d \alpha^{*}(u)}{d u}\right\| d u=|\mu| s(t) .
$$

In this section, we denote by a prime "' " the differentiation with respect to $s$. The $i^{\text {th }}$ curvature $\kappa_{i}$ of non-null curve $\alpha$ is given by

$$
\kappa_{i}=\mathbf{e}_{i}^{\prime} \cdot \mathbf{e}_{i+1}
$$

for $1 \leq i \leq n-1$ where $\kappa_{j}>0$ and $\kappa_{n-1} \neq 0, j=1,2, \ldots, n-2$. The Frenet-Serret equations of $\alpha$ in $\mathbb{E}_{1}^{n}$ is

$$
\begin{aligned}
\mathbf{e}_{1}^{\prime} & =\varepsilon_{2} \kappa_{1} \mathbf{e}_{2}, \\
\mathbf{e}_{i}^{\prime} & =-\varepsilon_{i-1} \kappa_{i-1} \mathbf{e}_{i-1}+\varepsilon_{i+1} \kappa_{i} \mathbf{e}_{i+1}, \quad \text { for } 2 \leq i \leq n-1 \\
\mathbf{e}_{n}^{\prime} & =-\varepsilon_{n-1} \kappa_{n-1} \mathbf{e}_{n-1},
\end{aligned}
$$

where

$$
\varepsilon_{\ell}=\left\{\begin{aligned}
-1 & \text { if } \mathbf{e}_{\ell} \text { is the timelike vector } \\
1 & \text { if } \mathbf{e}_{\ell} \text { is the spacelike vector }
\end{aligned} \quad \text { for } 1 \leq \ell \leq n\right.
$$

Let $\gamma(s)=\mathbf{e}_{1}(s)$ be the spherical tangent indicatrix of $\alpha$ and $\sigma$ be an arc length parameter of $\gamma$. It can be given a reparametrization of $\alpha$ by $\sigma$

$$
\alpha=\alpha(\sigma): I \rightarrow \mathbb{E}_{1}^{n},
$$

and the parameter $\sigma$ is called a spherical arc length parameter of $\alpha$. It is easily computed that

$$
d \sigma=\kappa_{1} d s \quad \text { and } \quad \frac{d \alpha}{d \sigma}=\frac{1}{\kappa_{1}} \mathbf{e}_{1}
$$

Thus, we can write

$$
\frac{d}{d \sigma}\left[\begin{array}{c}
\mathbf{e}_{1} \\
\mathbf{e}_{2} \\
\mathbf{e}_{3} \\
\vdots \\
\mathbf{e}_{n-1} \\
\mathbf{e}_{n}
\end{array}\right]=\left[\begin{array}{cccccc}
0 & \varepsilon_{2} & 0 & \cdots & 0 & 0 \\
-\varepsilon_{1} & 0 & \varepsilon_{3} \frac{\kappa_{2}}{\kappa_{1}} & \cdots & 0 & 0 \\
0 & -\varepsilon_{2} \frac{\kappa_{2}}{\kappa_{1}} & 0 & \cdots & 0 & 0 \\
\vdots & \vdots & \ddots & \ddots & \vdots & \vdots \\
0 & 0 & 0 & \cdots & 0 & \varepsilon_{n} \frac{\kappa_{n-1}}{\kappa_{1}} \\
0 & 0 & 0 & \cdots & -\varepsilon_{n-1} \frac{\kappa_{n-1}}{\kappa_{1}} & 0
\end{array}\right]\left[\begin{array}{c}
\mathbf{e}_{1} \\
\mathbf{e}_{2} \\
\mathbf{e}_{3} \\
\vdots \\
\mathbf{e}_{n-1} \\
\mathbf{e}_{n}
\end{array}\right]
$$

We consider the pseudo-orthogonal $n$-frame

$$
\left\{\frac{1}{\kappa_{1}} \mathbf{e}_{1}(\sigma), \frac{1}{\kappa_{1}} \mathbf{e}_{2}(\sigma), \ldots, \frac{1}{\kappa_{1}} \mathbf{e}_{n}(\sigma)\right\}, \quad \sigma \in I,
$$


for the curve given by (8). Let $\tilde{\kappa}_{1}$ denote the function $-\frac{1}{\kappa_{1}} \frac{d \kappa_{1}}{d \sigma}$ and $\tilde{\kappa}_{i}$ denote the function $\frac{\kappa_{i}}{\kappa_{1}}$ for $i=2,3, \ldots, n-1$. Then, using $(7),(9)$ and (10), we get

$$
\begin{aligned}
& \frac{d}{d \sigma}\left(\frac{1}{\kappa_{1}} \mathbf{e}_{1}(\sigma), \frac{1}{\kappa_{1}} \mathbf{e}_{2}(\sigma), \ldots, \frac{1}{\kappa_{1}} \mathbf{e}_{n}(\sigma)\right)^{T} \\
= & \tilde{K}\left(\frac{1}{\kappa_{1}} \mathbf{e}_{1}(\sigma), \frac{1}{\kappa_{1}} \mathbf{e}_{2}(\sigma), \ldots, \frac{1}{\kappa_{1}} \mathbf{e}_{n}(\sigma)\right)^{T},
\end{aligned}
$$

where

$$
\tilde{K}=\left[\begin{array}{cccccccc}
\tilde{\kappa}_{1} & \varepsilon_{2} & 0 & 0 & \cdots & 0 & 0 & 0 \\
-\varepsilon_{1} & \tilde{\kappa}_{1} & \varepsilon_{3} \tilde{\kappa}_{2} & 0 & \cdots & 0 & 0 & 0 \\
0 & -\varepsilon_{2} \tilde{\kappa}_{2} & \tilde{\kappa}_{1} & \varepsilon_{4} \tilde{\kappa}_{3} & \cdots & 0 & 0 & 0 \\
\vdots & \vdots & \ddots & \ddots & \ddots & \vdots & \vdots & \vdots \\
0 & 0 & 0 & 0 & \cdots & -\varepsilon_{n-2} \tilde{\kappa}_{n-2} & \tilde{\kappa}_{1} & \varepsilon_{n} \tilde{\kappa}_{n-1} \\
0 & 0 & 0 & 0 & \cdots & 0 & -\varepsilon_{n-1} \tilde{\kappa}_{n-1} & \tilde{\kappa}_{1}
\end{array}\right] .
$$

Proposition 3. The pseudo-orthogonal frame

$$
\frac{1}{\kappa_{1}} \mathbf{e}_{1}(\sigma), \frac{1}{\kappa_{1}} \mathbf{e}_{2}(\sigma), \ldots, \frac{1}{\kappa_{1}} \mathbf{e}_{n}(\sigma), \quad \sigma \in I
$$

and the functions $\tilde{\kappa}_{1}=-\frac{1}{\kappa_{1}} \frac{d \kappa_{1}}{d \sigma}, \tilde{\kappa}_{i}=\frac{\kappa_{i}}{\kappa_{1}}(i=2,3, \ldots, n-1)$ are invariant under the group of $\boldsymbol{S i m}^{+}\left(\mathbb{E}_{1}^{n}\right)$ of the Minkowski n-space for the non-null Frenet curve given by (8).

Proof. Let be $\mu>0$ when $n$ is odd and $\sigma^{*}$ be a spherical arc length parameter of $\alpha^{*}$. $\left\{\mathbf{e}_{1}^{*}=A\left(\mathbf{e}_{1}\right), \ldots, \mathbf{e}_{n}^{*}=A\left(\mathbf{e}_{n}\right)\right\}$ is the Frenet-Serret frame of $\alpha^{*}$. From (4), (5) and (6), the $i^{\text {th }}$ curvature $\kappa_{i}^{*}$ of non-null curve $\alpha^{*}$ can compute as follow

$$
\kappa_{i}^{*}\left(s^{*}\right)=\frac{d \mathbf{e}_{i}^{*}}{d s^{*}} \cdot \mathbf{e}_{i+1}^{*}=\frac{1}{\mu} \kappa_{i}(s) .
$$

We have

$$
d \sigma^{*}=\kappa_{1}^{*} d s^{*}=\kappa_{1} d s=d \sigma
$$

by using (13) and (9). Then, we get $\tilde{\kappa}_{1}^{*}=\tilde{\kappa}_{1}$ and $\tilde{\kappa}_{i}^{*}=\tilde{\kappa}_{i}$, i.e., the functions $\tilde{\kappa}_{i}$ are invariant under the p-similarity transformation. Also, by definition of the similarity transformation, we can write $\vec{f}\left(\frac{1}{\kappa_{1}} \mathbf{e}_{1}\right)=\frac{1}{\kappa_{1}^{*}} \mathbf{e}_{1}^{*}$. Thus, the pseudoorthogonal frame (12) is invariant under the orientation-preserving p-similarity transformation. This proof also is valid in the case of even $n$ and $\mu<0$.

Definition 4. Let $\alpha: I \rightarrow \mathbb{E}_{1}^{n}$ be non-null curve of the class $C^{n}$ parameterized by a spherical arc length parameter $\sigma$. The functions

$$
\tilde{\kappa}_{1}=-\frac{1}{\kappa_{1}} \frac{d \kappa_{1}}{d \sigma} \quad \text { and } \quad \tilde{\kappa}_{i}=\frac{\kappa_{i}}{\kappa_{1}}, \quad i=2,3, \ldots, n-1
$$

are called p-shape curvatures of $\alpha$ in $\mathbb{E}_{1}^{n}$. 
Remark 5. The equation (11) may be considered as the structure equation in $\mathbb{E}_{1}^{n}$ with respect to the pseudo-orthogonal frame (12) and the group $\mathbf{S i m}^{+}\left(\mathbb{E}_{1}^{n}\right)$.

\section{Fundamental theorem of a non-null curve in Lorentzian similarity geometry}

Two non-null space curves which have the same curvatures are always equivalent according to Lorentzian motion. This notion can be extended with respect to $\operatorname{Sim}\left(\mathbb{E}_{1}^{n}\right)$ for the non-null space curves which have the same p-shape curvatures in $\mathbb{E}_{1}^{n}$.

Theorem 6 (Uniqueness Theorem). Let $\alpha, \alpha^{*}: I \rightarrow \mathbb{E}_{1}^{n}$ be two non-null space curves of class $C^{n}$ parameterized by the same spherical arc length parameter $\sigma$ and have the same causal character, where $I \subset \mathbb{R}$ is an open interval. Suppose that $\alpha$ and $\alpha^{*}$ have the same $p$-shape curvatures $\tilde{\kappa}_{i}=\tilde{\kappa}_{i}^{*}$ for any $\sigma \in I, i=$ $1,2, \ldots, n-1$.

i) If $n$ is odd and $\alpha, \alpha^{*}$ are the timelike curves, there exists a $f \in \boldsymbol{S i m}^{-}\left(\mathbb{E}_{1}^{n}\right)$ such that $\alpha^{*}=f \circ \alpha$.

ii) If $n$ is odd and $\alpha, \alpha^{*}$ are the spacelike curves, there exists a $f \in \boldsymbol{S i m}^{+}\left(\mathbb{E}_{1}^{n}\right)$ such that $\alpha^{*}=f \circ \alpha$.

iii) If $n$ is even, there exists a $f \in \boldsymbol{S i m}^{+}\left(\mathbb{E}_{1}^{n}\right)$ such that $\alpha^{*}=f \circ \alpha$.

Proof. Let's the Lorentzian curvatures and an arc-length parameters of $\alpha$, $\alpha^{*}$ denoted by $\kappa_{i}, \kappa_{i}^{*}$ and $s, s^{*}$. Using the equality $\tilde{\kappa}_{1}=\tilde{\kappa}_{1}^{*}$ and $(15)$, we get $\kappa_{1}=\mu \kappa_{1}^{*}$ for some real constant $\mu>0$. Then, the equalities $\tilde{\kappa}_{i}=\tilde{\kappa}_{i}^{*}$ $(i=2,3, \ldots, n-1)$ imply $\kappa_{i}=\mu \kappa_{i}^{*}$. On the other hand, from (14) we can write $d s=\frac{1}{\mu} d s^{*}$.

We can choose any point $\sigma_{0} \in I$. There exists a Lorentzian motion $\varphi$ of $\mathbb{E}_{1}^{n}$ such that

$$
\varphi\left(\alpha\left(\sigma_{0}\right)\right)=\alpha^{*}\left(\sigma_{0}\right) \text { and } \varphi\left(\mathbf{e}_{i}\left(\sigma_{0}\right)\right)=-\varepsilon_{i} \mathbf{e}_{i}^{*}\left(\sigma_{0}\right) \text { for } i=1,2, \ldots, n .
$$

Let's consider the function $\Psi: I \rightarrow \mathbb{R}$ defined by

$$
\begin{aligned}
\Psi(\sigma)= & \left\|\varphi\left(\mathbf{e}_{1}(\sigma)\right)-\varepsilon_{1} \mathbf{e}_{1}^{*}(\sigma)\right\|^{2}+\left\|\varphi\left(\mathbf{e}_{2}(\sigma)\right)-\varepsilon_{2} \mathbf{e}_{2}^{*}(\sigma)\right\|^{2} \\
& +\cdots+\left\|\varphi\left(\mathbf{e}_{n}(\sigma)\right)-\varepsilon_{n} \mathbf{e}_{n}^{*}(\sigma)\right\|^{2} .
\end{aligned}
$$

Then

$$
\begin{aligned}
\frac{d \Psi}{d \sigma}=2 & \left(\frac{d}{d \sigma} \varphi\left(\mathbf{e}_{1}(\sigma)\right)-\varepsilon_{1} \frac{d}{d \sigma} \mathbf{e}_{1}^{*}(\sigma)\right) \cdot\left(\varphi\left(\mathbf{e}_{1}(\sigma)\right)-\varepsilon_{1} \mathbf{e}_{1}^{*}(\sigma)\right) \\
& +2\left(\frac{d}{d \sigma} \varphi\left(\mathbf{e}_{2}(\sigma)\right)-\varepsilon_{2} \frac{d}{d \sigma} \mathbf{e}_{2}^{*}(\sigma)\right) \cdot\left(\varphi\left(\mathbf{e}_{2}(\sigma)\right)-\varepsilon_{2} \mathbf{e}_{2}^{*}(\sigma)\right) \\
& +\cdots+2\left(\frac{d}{d \sigma} \varphi\left(\mathbf{e}_{n}(\sigma)\right)-\varepsilon_{n} \frac{d}{d \sigma} \mathbf{e}_{3}^{*}(\sigma)\right) \cdot\left(\varphi\left(\mathbf{e}_{n}(\sigma)\right)-\varepsilon_{n} \mathbf{e}_{n}^{*}(\sigma)\right) .
\end{aligned}
$$


Using $\left\|\varphi\left(\mathbf{e}_{i}\right)\right\|^{2}=\left\|\mathbf{e}_{i}\right\|^{2}=\left\|\mathbf{e}_{i}^{*}\right\|^{2}=1$ we can write

$$
\begin{aligned}
\frac{d \Psi}{d \sigma}= & -2 \varepsilon_{1}\left[\left(\varphi\left(\frac{d}{d \sigma} \mathbf{e}_{1}\right)\right) \cdot \mathbf{e}_{1}^{*}+\varphi\left(\mathbf{e}_{1}\right) \cdot\left(\frac{d}{d \sigma} \mathbf{e}_{1}^{*}\right)\right] \\
& -2 \varepsilon_{2}\left[\left(\varphi\left(\frac{d}{d \sigma} \mathbf{e}_{2}\right)\right) \cdot \mathbf{e}_{2}^{*}+\varphi\left(\mathbf{e}_{2}\right) \cdot\left(\frac{d}{d \sigma} \mathbf{e}_{2}^{*}\right)\right] \\
& -\cdots-2 \varepsilon_{n}\left[\left(\varphi\left(\frac{d}{d \sigma} \mathbf{e}_{n}\right)\right) \cdot \mathbf{e}_{n}^{*}+\varphi\left(\mathbf{e}_{n}\right) \cdot\left(\frac{d}{d \sigma} \mathbf{e}_{n}^{*}\right)\right] .
\end{aligned}
$$

From (10), we get

$$
\begin{aligned}
\frac{d \Psi}{d \sigma}= & \left(-2 \varepsilon_{1} \varepsilon_{2}+2 \varepsilon_{2} \varepsilon_{1}\right)\left[\varphi\left(\mathbf{e}_{2}\right) \cdot \mathbf{e}_{1}^{*}\right]+\left(-2 \varepsilon_{1} \varepsilon_{2}+2 \varepsilon_{2} \varepsilon_{1}\right)\left[\varphi\left(\mathbf{e}_{1}\right) \cdot \mathbf{e}_{2}^{*}\right] \\
& +\left(-2 \varepsilon_{2} \varepsilon_{3} \tilde{\kappa}_{2}+2 \varepsilon_{3} \varepsilon_{2} \tilde{\kappa}_{2}^{*}\right)\left[\varphi\left(\mathbf{e}_{3}\right) \cdot \mathbf{e}_{2}^{*}\right]+\left(-2 \varepsilon_{2} \varepsilon_{3} \tilde{\kappa}_{2}^{*}+2 \varepsilon_{2} \varepsilon_{3} \tilde{\kappa}_{2}\right)\left[\varphi\left(\mathbf{e}_{2}\right) \cdot \mathbf{e}_{3}^{*}\right] \\
& +\cdots+\left(2 \varepsilon_{n-1} \varepsilon_{n} \tilde{\kappa}_{n-1}-2 \varepsilon_{n-1} \varepsilon_{n} \tilde{\kappa}_{n-1}^{*}\right)\left[\varphi\left(\mathbf{e}_{n-1}\right) \cdot \mathbf{e}_{n}^{*}\right] \\
& +\left(2 \varepsilon_{n-1} \varepsilon_{n} \tilde{\kappa}_{n-1}^{*}-2 \varepsilon_{n-1} \varepsilon_{n} \tilde{\kappa}_{n-1}\right)\left[\varphi\left(\mathbf{e}_{n}\right) \cdot \mathbf{e}_{n-1}^{*}\right] .
\end{aligned}
$$

Since we have $\tilde{\kappa}_{i}=\tilde{\kappa}_{i}^{*}$, we find $\frac{d \Psi}{d \sigma}=0$ for any $\sigma \in I$. On the other hand, we know $\Psi\left(\sigma_{0}\right)=0$ and thus we can write $\Psi(\sigma)=0$ for any $\sigma \in I$. As a result, we can say that

$$
\varphi\left(\mathbf{e}_{i}(\sigma)\right)=\varepsilon_{i} \mathbf{e}_{i}^{*}(\sigma), \quad \forall \sigma \in I, \quad i=1,2, \ldots, n .
$$

The map $g=\mu \varphi: \mathbb{E}_{1}^{n} \rightarrow \mathbb{E}_{1}^{n}$ is a p-similarity of $\mathbb{E}_{1}^{n}$. We examine an other function $\Phi: I \rightarrow \mathbb{R}$ such that

$$
\Phi(\sigma)=\left\|\frac{d}{d \sigma} g(\alpha(\sigma))-\varepsilon_{1} \frac{d}{d \sigma} \alpha^{*}(\sigma)\right\|^{2} \quad \text { for } \forall \sigma \in I .
$$

Taking derivative of this function with respect to $\sigma$ we get

$$
\begin{aligned}
\frac{d \Phi}{d \sigma}= & 2 g\left(\frac{d^{2} \alpha}{d \sigma^{2}}\right) \cdot g\left(\frac{d \alpha}{d \sigma}\right)-2 \varepsilon_{1}\left[g\left(\frac{d^{2} \alpha}{d \sigma^{2}}\right) \cdot \frac{d \alpha^{*}}{d \sigma}\right] \\
& -\varepsilon_{1} 2 \frac{d^{2} \alpha^{*}}{d \sigma^{2}} \cdot g\left(\frac{d \alpha}{d \sigma}\right)+2\left[\frac{d^{2} \alpha^{*}}{d \sigma^{2}} \cdot \frac{d \alpha^{*}}{d \sigma}\right] .
\end{aligned}
$$

Since the function $\varphi$ is linear map, we can write by (9) and (16) the following equation

$$
\frac{d \Phi}{d \sigma}=2 \varepsilon_{1}^{*} \mu^{2} \frac{\tilde{\kappa}_{1}}{\kappa_{1}^{2}}-2 \varepsilon_{1}^{*} \mu \frac{\tilde{\kappa}_{1}}{\kappa_{1} \kappa_{1}^{*}}-2 \varepsilon_{1}^{*} \mu \frac{\tilde{\kappa}_{1}^{*}}{\kappa_{1} \kappa_{1}^{*}}+2 \varepsilon_{1}^{*} \frac{\tilde{\kappa}_{1}^{*}}{\left(\kappa_{1}^{*}\right)^{2}} .
$$

Using $\mu=\frac{\kappa_{1}}{\kappa_{1}^{*}}$ it is obtained $\frac{d \Phi}{d \sigma}=0$. Also, it can be found

$$
\frac{d}{d \sigma} g\left(\alpha\left(\sigma_{0}\right)\right)=g\left(\frac{1}{\kappa} \mathbf{e}_{1}\left(\sigma_{0}\right)\right)=\varepsilon_{1} \frac{1}{\kappa^{*}} \mathbf{e}_{1}^{*}\left(\sigma_{0}\right)
$$


Then, we conclude that $\Phi\left(\sigma_{0}\right)=0$ from the equation $\frac{d}{d \sigma} \alpha^{*}\left(\sigma_{0}\right)=\frac{1}{\kappa^{*}} \mathbf{e}_{1}^{*}\left(\sigma_{0}\right)$. Hence, it can be said $\Phi(\sigma)=0$ for $\forall \sigma \in I$. This means that

$$
\frac{d}{d \sigma} g(\alpha(\sigma))=\varepsilon_{1} \frac{d}{d \sigma} \alpha^{*}(\sigma)
$$

or equivalently $\alpha^{*}(\sigma)=\varepsilon_{1} g(\alpha(\sigma))+\mathbf{b}$ where $\mathbf{b}$ is a constant vector. Then, the image of $\alpha$ under the p-similarity $f=\xi \circ\left(\varepsilon_{1} g\right)$ is the non-null curve $\alpha^{*}$, where $\xi: \mathbb{E}_{1}^{n} \rightarrow \mathbb{E}_{1}^{n}$ is a translation function determined by $\mathbf{b}$. We consider that $n$ is odd. If the curves $\alpha, \alpha^{*}$ are taken as the timelike curve, then the p-similarity transformation $f$ is an orientation-reversing transformation. Also, when the curves $\alpha, \alpha^{*}$ are the spacelike curves, p-similarity transformation $f$ is an orientation-preserving transformation.

The following theorem show that every $n-1$ functions of class $C^{\infty}$ according to a p-similarity determine a non-null Frenet curve under some initial conditions.

Theorem 7 (Existence Theorem). Let $z_{i}: I \rightarrow \mathbb{R}, i=1,2, \ldots, n-1$, be functions of class $C^{\infty}$ such that $z_{1}, z_{2}, \ldots, z_{n-1}$ have the same sign and $\mathbf{e}_{1}^{0}$, $\mathbf{e}_{2}^{0}, \ldots, \mathbf{e}_{n}^{0}$ be a pseodo-orthonormal $n$-frame at a point $x_{0}$ in the Minkowski $n$ space. According to a p-similarity with center $x_{0}$ there exists a unique non-null space curve $\alpha: I \rightarrow \mathbb{E}_{1}^{n}$ parameterized by a spherical arc-length parameter such that $\alpha$ satisfies the following conditions:

(i) There exists $\sigma_{0} \in I$ such that $\alpha\left(\sigma_{0}\right)=x_{0}$ and the Frenet-Serret $n$-frame of $\alpha$ at $x_{0}$ is $\mathbf{e}_{1}^{0}, \mathbf{e}_{2}^{0}, \ldots, \mathbf{e}_{n}^{0}$.

(ii) $\tilde{\kappa}_{i}(\sigma)=z_{i}(\sigma)$ for any $\sigma \in I$ and $i=1,2, \ldots, n-1$.

Proof. Let us consider the following system of differential equations with respect to a matrix-valued function $\mathbf{W}(\sigma)=\left(\mathbf{e}_{1}, \mathbf{e}_{2}, \ldots, \mathbf{e}_{n}\right)^{T}$

$$
\frac{d \mathbf{W}}{d \sigma}(\sigma)=\mathbf{M}(\sigma) \mathbf{W}(\sigma)
$$

with a given matrix

$$
\mathbf{M}(\sigma)=\left[\begin{array}{ccccccc}
0 & \varepsilon_{2} & 0 & \cdots & 0 & 0 & 0 \\
-\varepsilon_{1} & 0 & \varepsilon_{3} z_{2} & \cdots & 0 & 0 & 0 \\
0 & -\varepsilon_{2} z_{2} & 0 & \ddots & 0 & 0 & 0 \\
0 & 0 & -\varepsilon_{3} z_{3} & \ddots & 0 & 0 & 0 \\
\vdots & \vdots & \vdots & \ddots & \ddots & \vdots & \vdots \\
0 & 0 & 0 & \cdots & -\varepsilon_{n-2} z_{n-2} & 0 & \varepsilon_{n} z_{n-1} \\
0 & 0 & 0 & \cdots & 0 & -\varepsilon_{n-1} z_{n-1} & 0
\end{array}\right] .
$$

The system (17) has a unique solution $\mathbf{W}(\sigma)$ which satisfies the initial conditions $\mathbf{W}\left(\sigma_{0}\right)=\left(\mathbf{e}_{1}^{0}, \mathbf{e}_{2}^{0}, \ldots, \mathbf{e}_{n}^{0}\right)^{T}$ for $\sigma_{0} \in I$. If $\mathbf{W}^{t}(\sigma)$ is the transposed 
matrix of $\mathbf{W}(\sigma)$, then

$$
\begin{aligned}
\frac{d}{d \sigma}\left(\mathbf{I}^{*} \mathbf{W}^{t} \mathbf{I}^{*} \mathbf{W}\right) & =\mathbf{I}^{*} \frac{d}{d \sigma} \mathbf{W}^{t} \mathbf{I}^{*} \mathbf{W}+\mathbf{I}^{*} \mathbf{W}^{t} \mathbf{I}^{*} \frac{d}{d \sigma} \mathbf{W} \\
& =\mathbf{I}^{*} \mathbf{W}^{t} \mathbf{M}^{t} \mathbf{I}^{*} \mathbf{W}+\mathbf{I}^{*} \mathbf{W}^{t} \mathbf{I}^{*} \mathbf{M} \mathbf{W} \\
& =\mathbf{I}^{*} \mathbf{W}^{t}\left(\mathbf{M}^{t} \mathbf{I}^{*}+\mathbf{I}^{*} \mathbf{M}\right) \mathbf{W}=0
\end{aligned}
$$

because of the equation $\mathbf{M}^{t} \mathbf{I}^{*}+\mathbf{I}^{*} \mathbf{M}=[0]_{n \times n}$ where $\mathbf{I}^{*}=\operatorname{diag}\left(\varepsilon_{1}, \varepsilon_{2}, \ldots, \varepsilon_{n}\right)$. Also, we have $\mathbf{I}^{*} \mathbf{W}^{t}\left(\sigma_{0}\right) \mathbf{I}^{*} \mathbf{W}\left(\sigma_{0}\right)=\mathbf{I}$ where $\mathbf{I}$ is the unit matrix since $\left\{\mathbf{e}_{1}^{0}, \mathbf{e}_{2}^{0}\right.$, $\left.\ldots, \mathbf{e}_{n}^{0}\right\}$ is the pseudo-orthonormal $n$-frame. As a result, we find

$$
\mathbf{I}^{*} \mathbf{X}^{t}(\sigma) \mathbf{I}^{*} \mathbf{X}(\sigma)=\mathbf{I}
$$

for any $\sigma \in I$. It means that the vector fields $\left\{\mathbf{e}_{1}^{0}, \mathbf{e}_{2}^{0}, \ldots, \mathbf{e}_{n}^{0}\right\}$ form pseudoorthonormal frame field.

Let $\alpha: I \rightarrow \mathbb{E}_{1}^{n}$ be the regular non-null curve given by

$$
\alpha(\sigma)=x_{0}+\int_{\sigma_{0}}^{\sigma} e^{\int z_{1}(\sigma) d \sigma} \mathbf{e}_{1}(\sigma) d \sigma, \quad \sigma \in I .
$$

By the equality (17) and the linear independence of $\left\{\mathbf{e}_{1}, \mathbf{e}_{2}, \ldots, \mathbf{e}_{n}\right\}$, we get that $\alpha(\sigma)$ is a non-null space curve in $\mathbb{E}_{1}^{n}$ with p-shape curvatures $\tilde{\kappa}_{i}(\sigma)=$ $z_{i}(\sigma)$ for $i=1,2, \ldots, n-1$. Also, the pseudo-orthonormal n-frame $\mathbf{e}_{1}(\sigma)$, $\mathbf{e}_{2}(\sigma), \ldots, \mathbf{e}_{n}(\sigma)$ is a Frenet-Serret n-frame of the non-null curve $\alpha$.

By Theorems 6 and 7, we get the following theorem.

Theorem 8. Let $z_{i}: I \rightarrow \mathbb{R}, i=1,2, \ldots, n-1$, be the functions of class $C^{\infty}$. According to p-similarity there exists a unique non-null space curve with p-shape curvatures $z_{i}$.

Example 9. Let p-shape curvatures $\left(\tilde{\kappa}_{1}, \tilde{\kappa}_{2}\right)$ of the $\alpha: I \rightarrow \mathbb{E}_{1}^{3}$ be $(0, a)$, where $a \neq 0$ is real constant, and the unit vector $\mathbf{e}_{2}(\sigma)$ be a timelike vector. Choose initial conditions

$$
\mathbf{e}_{1}^{0}=\left(0,-\frac{1}{\sqrt{1+a^{2}}}, \frac{a}{\sqrt{1+a^{2}}}\right), \mathbf{e}_{2}^{0}=(1,0,0), \mathbf{e}_{3}^{0}=\left(0, \frac{a}{\sqrt{1+a^{2}}}, \frac{1}{\sqrt{1+a^{2}}}\right) .
$$

Then, the system (17) describes a spacelike vector $\mathbf{e}_{1}$ defined by

$$
\mathbf{e}_{1}(\sigma)=\left(\frac{1}{\sqrt{1+a^{2}}} \sinh \left(\sqrt{1+a^{2}} \sigma\right),-\frac{1}{\sqrt{1+a^{2}}} \cosh \left(\sqrt{1+a^{2}} \sigma\right), \frac{a}{\sqrt{1+a^{2}}}\right)
$$

with $\mathbf{e}_{1}(0)=\mathbf{e}_{1}^{0}$, in the Minkowski 3-space. Solving the equation (18) we obtain the spacelike Frenet curve $\alpha$ (see Figure 1) parameterized by

$$
\begin{aligned}
\alpha(\sigma)= & \left(\frac{1}{1+a^{2}} \cosh \left(\sqrt{1+a^{2}} \sigma\right),-\frac{1}{1+a^{2}} \sinh \left(\sqrt{1+a^{2}} \sigma\right), \frac{a}{\sqrt{1+a^{2}}} \sigma\right), \\
& \sigma \in I .
\end{aligned}
$$




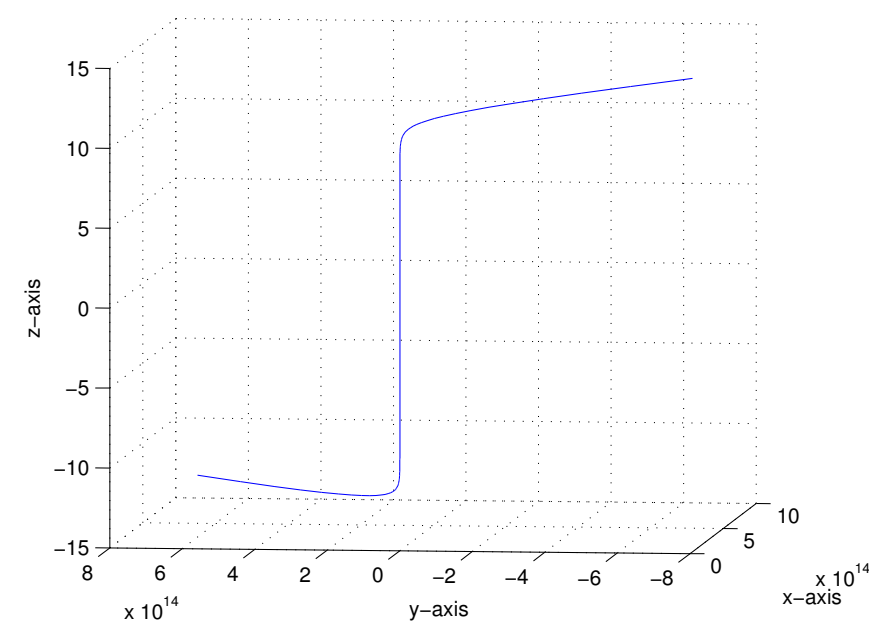

FIGURE 1. The spacelike curve parameterized by (21) for $\mathrm{a}=1$

Example 10. Let p-shape curvatures $\left(\tilde{\kappa}_{1}, \tilde{\kappa}_{2}, \tilde{\kappa}_{3}\right)$ of the $\alpha: I \rightarrow \mathbb{E}_{1}^{4}$ be $\left(\frac{1}{\sigma}, 0,0\right)$ and the unit vector $\mathbf{e}_{1}(\sigma)$ be a timelike vector. Choose initial conditions

$$
\begin{array}{r}
\mathbf{e}_{1}^{0}=(\sqrt{2}, 0,1,0), \mathbf{e}_{2}^{0}=\left(0, \frac{1}{\sqrt{2}}, 0, \frac{1}{\sqrt{2}}\right), \\
\mathbf{e}_{3}^{0}=(1,0, \sqrt{2}, 0), \mathbf{e}_{4}^{0}=\left(0,-\frac{1}{\sqrt{2}}, 0, \frac{1}{\sqrt{2}}\right) .
\end{array}
$$

Then, the system (17) describes a timelike vector $\mathbf{e}_{1}$ defined by

$$
\mathbf{e}_{1}(\sigma)=\left(\sqrt{2} \cosh \sigma, \frac{\sinh \sigma}{\sqrt{2}}, \cosh \sigma, \frac{\sinh \sigma}{\sqrt{2}}\right)
$$

with $\mathbf{e}_{1}(0)=\mathbf{e}_{1}^{0}$, in the Minkowski space-time $\mathbb{E}_{1}^{4}$. Solving the equation (18) we obtain the timelike Frenet curve parameterized by

$$
\begin{gathered}
\alpha(\sigma)=\left(\sqrt{2}(\sigma \sinh \sigma-\cosh \sigma), \frac{1}{\sqrt{2}}(\sigma \cosh \sigma-\sinh \sigma),\right. \\
\left.\sigma \sinh \sigma-\cosh \sigma, \frac{1}{\sqrt{2}}(\sigma \cosh \sigma-\sinh \sigma)\right)
\end{gathered}
$$

for any $\sigma \in I$, where $\sigma$ is a spherical arc-length parametrization of $\alpha$.

\section{The relation between focal curvatures and p-shape curvatures}

Let $\alpha: I \rightarrow \mathbb{E}_{1}^{n}$ be a unit speed non-null space curve with the Frenet frame $\mathbf{e}_{1}, \mathbf{e}_{2}, \ldots, \mathbf{e}_{n}$ and let $s$ be an arc length parameter of $\alpha$. The curve $\gamma: I \rightarrow \mathbb{E}_{1}^{n}$ 
consisting of the centers of the osculating hyperspheres of the curve $\alpha$ is called the focal curve of $\alpha$. The focal curve can be represented by

$$
\gamma(s)=\alpha(s)+m_{1}(s) \mathbf{e}_{2}+m_{2}(s) \mathbf{e}_{3}+\cdots+m_{n-1}(s) \mathbf{e}_{n},
$$

where $m_{1}, \ldots, m_{n-1}$ are smooth functions called focal curvatures of $\alpha$. Then, we have the following theorem from [27].

Theorem 11. The Euclidean curvatures of a non-null space curve $\alpha$ in $\mathbb{E}_{1}^{n}$, parameterized by arc length, are given in terms of the focal curvatures of $\alpha$ by the formula:

$$
\kappa_{1}=\frac{\varepsilon_{1}}{m_{1}}, \quad \kappa_{i}=\frac{\varepsilon_{2} m_{1} m_{1}^{\prime}+\varepsilon_{3} m_{2} m_{2}^{\prime}+\cdots+\varepsilon_{i} m_{i-1} m_{i-1}^{\prime}}{m_{i-1} m_{i}}
$$

for $2 \leq i \leq n$.

Now, we can restate all the p-shape curvatures $\tilde{\kappa}_{i}$ via the focal curvatures and their derivatives.

Proposition 12. Let $\alpha: I \rightarrow \mathbb{E}_{1}^{n}$ be a unit speed non-null space curve whose all Euclidean curvatures are non-zero. Then

$$
\tilde{\kappa}_{1}=\varepsilon_{1} m_{1}^{\prime}, \quad \tilde{\kappa}_{i}=\frac{\varepsilon_{1} m_{1}}{m_{i-1} m_{i}}\left(\varepsilon_{2} m_{1} m_{1}^{\prime}+\varepsilon_{3} m_{2} m_{2}^{\prime}+\cdots+\varepsilon_{i} m_{i-1} m_{i-1}^{\prime}\right)
$$

for $2 \leq i \leq n$.

Proof. By (15) we know

$$
\tilde{\kappa}_{1}=\left(\frac{1}{\kappa_{1}}\right)^{\prime} \quad \text { and } \quad \tilde{\kappa}_{i}=\frac{\kappa_{i}}{\kappa_{1}}, \quad i=2,3, \ldots, n-1 .
$$

So, It can be easily found the equations (22) by using Theorem 11 and (23).

\section{The non-null self-similar space curves}

In this section, we study non-null self-similar space curves in the Minkowski n-space $\mathbb{E}_{1}^{n}$. A non-null space curve $\alpha: I \rightarrow \mathbb{E}_{1}^{n}$ is called self-similar if any p-similarity $f \in G$ conserve globally $\alpha$ and $G$ acts transitively on $\alpha$ where $G$ is a one-parameter subgroup of $\operatorname{Sim}\left(\mathbb{E}_{1}^{n}\right)$. This means that all its the p-shape curvatures $\tilde{\kappa}_{1}, \tilde{\kappa}_{2}, \ldots, \tilde{\kappa}_{n-1}$ are constant. In fact, let $p=\alpha\left(s_{1}\right)$ and $q=\alpha\left(s_{2}\right)$ be two different points lying on $\alpha$. Since $G$ acts transitively on $\alpha$, there is a similarity $f \in G$ such that $f(p)=q$ and then $\tilde{\kappa}_{i}\left(s_{1}\right)=\tilde{\kappa}_{i}\left(s_{2}\right)$ because of the invariance of $\tilde{\kappa}_{i}, i=1,2, \ldots, n-1$. Every non-null self-similar curve with the constant invariants $\tilde{\kappa}_{1}=0, \tilde{\kappa}_{i}>0$ and $\tilde{\kappa}_{n-1} \neq 0, i=2,3, \ldots, n-2$, has the constant Euclidean curvatures

$$
\kappa_{1}>0, \kappa_{2}=\tilde{\kappa}_{2} \kappa_{1}>0, \ldots, \kappa_{n-2}=\tilde{\kappa}_{n-2} \kappa_{1}>0, \kappa_{n-1}=\tilde{\kappa}_{n-1} \kappa_{1} \neq 0 .
$$

We will investigate the non-null space curve with the constant p-shape curvatures

$$
\tilde{\kappa}_{1} \neq 0, \tilde{\kappa}_{2} \neq 0, \ldots, \tilde{\kappa}_{n-1} \neq 0
$$


Consider the constant matrix

$$
M=\left[\begin{array}{cccccccc}
0 & \varepsilon_{2} & 0 & 0 & \cdots & 0 & 0 & 0 \\
-\varepsilon_{1} & 0 & \varepsilon_{3} \tilde{\kappa}_{2} & 0 & \cdots & 0 & 0 & 0 \\
0 & -\varepsilon_{2} \tilde{\kappa}_{2} & 0 & \varepsilon_{4} \tilde{\kappa}_{3} & \cdots & 0 & 0 & 0 \\
0 & 0 & -\varepsilon_{3} \tilde{\kappa}_{3} & 0 & \ddots & 0 & 0 & 0 \\
\vdots & \vdots & \vdots & \ddots & \ddots & \vdots & \vdots & \vdots \\
0 & 0 & 0 & 0 & \cdots & -\varepsilon_{n-2} \tilde{\kappa}_{n-2} & 0 & \varepsilon_{n} \tilde{\kappa}_{n-1} \\
0 & 0 & 0 & 0 & \cdots & 0 & -\varepsilon_{n-1} \tilde{\kappa}_{n-1} & 0
\end{array}\right] .
$$

The matrix $M^{2}$ is a semi skew-symmetrix matrix and has exactly $k=\left\lfloor\frac{n}{2}\right\rfloor$ eigenvalues with multiplicity two: $\lambda_{1}^{2}, \lambda_{2}^{2}, \ldots, \lambda_{k}^{2}$. According to Corollary 3.3 in [29], the normal form of the matrix $M$ is either

$$
\left[\begin{array}{ccccccc}
0 & \varepsilon_{2} \lambda_{1} & 0 & 0 & \cdots & 0 & 0 \\
-\varepsilon_{1} \lambda_{1} & 0 & 0 & 0 & \cdots & 0 & 0 \\
0 & 0 & 0 & \varepsilon_{4} \lambda_{2} & \cdots & 0 & 0 \\
0 & 0 & -\varepsilon_{3} \lambda_{2} & 0 & \ddots & 0 & 0 \\
\vdots & \vdots & \vdots & \ddots & \ddots & \vdots & \vdots \\
0 & 0 & 0 & 0 & \cdots & 0 & \varepsilon_{n} \lambda_{k} \\
0 & 0 & 0 & 0 & \cdots & -\varepsilon_{n-1} \lambda_{k} & 0
\end{array}\right]
$$

in the case of even $n$, or the same matrix with an additional row (column) of zeros in the case of odd $n$.

\subsection{Non-null self-similar curves in even-dimensional Minkowski space}

Any non-null self-similar curve in $\mathbb{E}_{1}^{2 k}$ can be described by its constant pshape curvatures with respect to Theorem 7. Let's see this via the following theorem.

Theorem 13. Let $\alpha: I \rightarrow \mathbb{E}_{1}^{2 k}$ be a non-null self-similar curve with the constant $p$-shape curvatures $\tilde{\kappa}_{1} \neq 0, \tilde{\kappa}_{2} \neq 0, \ldots, \tilde{\kappa}_{2 k-1} \neq 0$. Suppose that $\lambda_{1}^{2}$, $\lambda_{2}^{2}, \ldots, \lambda_{k}^{2}$ are all different eigenvalues of the symmetric matrix $M^{2}$. So,

i) If the unit vector $\mathbf{e}_{1}$ is a timelike vector, then a parametric statement of the timelike self-similar curve $\alpha$ according to arc-length parameter $\sigma$ can be written in the form

$$
\begin{aligned}
\alpha(\sigma)=( & \frac{a_{1}}{b_{1}} e^{\tilde{\kappa}_{1} \sigma} \sinh \theta_{1}, \frac{a_{1}}{b_{1}} e^{\tilde{\kappa}_{1} \sigma} \cosh \theta_{1}, \frac{a_{2}}{b_{2}} e^{\tilde{\kappa}_{1} \sigma} \sin \theta_{2}, \\
& \left.-\frac{a_{2}}{b_{2}} e^{\tilde{\kappa}_{1} \sigma} \cos \theta_{2}, \ldots, \frac{a_{k}}{b_{k}} e^{\tilde{\kappa}_{1} \sigma} \sin \theta_{k},-\frac{a_{k}}{b_{k}} e^{\tilde{\kappa}_{1} \sigma} \cos \theta_{k}\right),
\end{aligned}
$$

where

$$
b_{1}=\sqrt{\lambda_{1}^{2}-\tilde{\kappa}_{1}^{2}}, \quad \theta_{1}=\lambda_{1} \sigma-\cosh ^{-1}\left(\frac{\lambda_{1}}{\sqrt{\lambda_{1}^{2}-\tilde{\kappa}_{1}^{2}}}\right)
$$


2084

MUSTAFA ÖZDEMIR AND HAKAN SIMSEK

and for $i=2, \ldots, k$

$$
b_{i}=\sqrt{\lambda_{i}^{2}+\tilde{\kappa}_{1}^{2}}, \quad \theta_{i}=\lambda_{i} \sigma+\cos ^{-1}\left(\frac{\lambda_{i}}{\sqrt{\lambda_{i}^{2}+\tilde{\kappa}_{1}^{2}}}\right) .
$$

The real different non-zero numbers $a_{1}, \ldots, a_{k}$ are a solution of the system of $k$ algebraic quadratic equations

$$
\mathbf{e}_{1} \cdot \mathbf{e}_{1}=-1 \quad \text { and } \quad \mathbf{e}_{i} \cdot \mathbf{e}_{i}=1, \quad i=2, \ldots, k,
$$

determined by the vectors

$$
\begin{aligned}
\mathbf{e}_{1}(\sigma) & =e^{-\tilde{\kappa}_{1} \sigma} \frac{d}{d \sigma} \alpha(\sigma), \\
\mathbf{e}_{2}(\sigma) & =\frac{d}{d \sigma} \mathbf{e}_{1}(\sigma), \\
\mathbf{e}_{3}(\sigma) & =\frac{1}{\tilde{\kappa}_{2}}\left(-\mathbf{e}_{1}(\sigma)+\frac{d}{d \sigma} \mathbf{e}_{2}(\sigma)\right), \\
\mathbf{e}_{4}(\sigma) & =\frac{1}{\tilde{\kappa}_{3}}\left(\tilde{\kappa}_{2} \mathbf{e}_{2}(\sigma)+\frac{d}{d \sigma} \mathbf{e}_{3}(\sigma)\right), \\
& \vdots \\
\mathbf{e}_{k}(\sigma) & =\frac{1}{\tilde{\kappa}_{k-1}}\left(\tilde{\kappa}_{k-2} \mathbf{e}_{k-2}(\sigma)+\frac{d}{d \sigma} \mathbf{e}_{k-1}(\sigma)\right) .
\end{aligned}
$$

ii) If the unit vector $\mathbf{e}_{2}$ is a timelike vector, then a parametric representation of the spacelike self-similar curve $\alpha$ according to arc-length parameter $\sigma$ can be written in the form

$$
\begin{aligned}
\alpha(\sigma)=( & -\frac{a_{1}}{b_{1}} e^{\tilde{\kappa}_{1} \sigma} \cosh \theta_{1},-\frac{a_{1}}{b_{1}} e^{\tilde{\kappa}_{1} \sigma} \sinh \theta_{1}, \frac{a_{2}}{b_{2}} e^{\tilde{\kappa}_{1} \sigma} \sin \theta_{2}, \\
& \left.-\frac{a_{2}}{b_{2}} e^{\tilde{\kappa}_{1} \sigma} \cos \theta_{2}, \ldots, \frac{a_{k}}{b_{k}} e^{\tilde{\kappa}_{1} \sigma} \sin \theta_{k},-\frac{a_{k}}{b_{k}} e^{\tilde{\kappa}_{1} \sigma} \cos \theta_{k}\right) .
\end{aligned}
$$

The real different non-zero numbers $a_{1}, \ldots, a_{k}$ are a solution of the system of $k$ algebraic quadratic equations

$$
\mathbf{e}_{2} \cdot \mathbf{e}_{2}=-1 \quad \text { and } \quad \mathbf{e}_{i} \cdot \mathbf{e}_{i}=1, \quad i=1,3,4, \ldots, k,
$$

determined by the vectors

$$
\begin{aligned}
& \mathbf{e}_{1}(\sigma)=e^{-\tilde{\kappa}_{1} \sigma} \frac{d}{d \sigma} \alpha(\sigma), \\
& \mathbf{e}_{2}(\sigma)=-\frac{d}{d \sigma} \mathbf{e}_{1}(\sigma), \\
& \mathbf{e}_{3}(\sigma)=\frac{1}{\tilde{\kappa}_{2}}\left(\mathbf{e}_{1}(\sigma)+\frac{d}{d \sigma} \mathbf{e}_{2}(\sigma)\right), \\
& \mathbf{e}_{4}(\sigma)=\frac{1}{\tilde{\kappa}_{3}}\left(-\tilde{\kappa}_{2} \mathbf{e}_{2}(\sigma)+\frac{d}{d \sigma} \mathbf{e}_{3}(\sigma)\right),
\end{aligned}
$$




$$
\mathbf{e}_{k}(\sigma)=\frac{1}{\tilde{\kappa}_{k-1}}\left(\tilde{\kappa}_{k-2} \mathbf{e}_{k-2}(\sigma)+\frac{d}{d \sigma} \mathbf{e}_{k-1}(\sigma)\right) .
$$

Proof. i) The systems of unit vectors $\mathbf{e}_{1}(\sigma), \mathbf{e}_{2}(\sigma), \ldots, \mathbf{e}_{2 k-1}(\sigma), \mathbf{e}_{2 k}(\sigma)$ can be expressed as a solution of the ordinary differential equations

$$
\frac{d}{d \sigma} \omega=M \omega
$$

where $\omega(\sigma)=\left(\mathbf{e}_{1}(\sigma), \mathbf{e}_{2}(\sigma), \ldots, \mathbf{e}_{2 k}(\sigma)\right)^{T}$. Using the normal form of the matrix $M$, we have that the unit vector $\mathbf{e}_{1}$ is the equal to

$$
\begin{gathered}
\mathbf{e}_{1}(\sigma)=\left(a_{1} \cosh \left(\lambda_{1} \sigma\right), a_{1} \sinh \left(\lambda_{1} \sigma\right), a_{2} \cos \left(\lambda_{2} \sigma\right), a_{2} \sin \left(\lambda_{2} \sigma\right), \ldots,\right. \\
\left.a_{k} \cos \left(\lambda_{k} \sigma\right), a_{k} \sin \left(\lambda_{k} \sigma\right)\right),
\end{gathered}
$$

where $a_{i}$ 's are a real constants satisfying $-a_{1}^{2}+\sum_{i=2}^{k} a_{i}^{2}=-1$.

If $X=\left(\alpha_{1}(\sigma), \alpha_{2}(\sigma), \ldots, \alpha_{2 k}(\sigma)\right)$ is considered as the parametric equation of the timelike curve $\alpha$, we get the following equation by (9)

$$
\frac{d}{d \sigma} X=\frac{1}{\kappa_{1}} \mathbf{e}_{1}
$$

where we have $\kappa_{1}=e^{-\tilde{\kappa}_{1} \sigma}$ by $\tilde{\kappa}_{1}=-\frac{1}{\kappa_{1}} \frac{d \kappa_{1}}{d \sigma}$. It can be obtained the following equation by (29)

$$
\begin{aligned}
& \alpha_{1}=\frac{a_{1}}{\tilde{\kappa}_{1}} e^{\tilde{\kappa}_{1} \sigma} \cosh \left(\lambda_{1} \sigma\right)-\frac{\lambda_{1}}{\tilde{\kappa}_{1}} \alpha_{2}, \\
& \alpha_{2}=\frac{a_{1}}{\tilde{\kappa}_{1}} e^{\tilde{\kappa}_{1} \sigma} \sinh \left(\lambda_{1} \sigma\right)-\frac{\lambda_{1}}{\tilde{\kappa}_{1}} \alpha_{1},
\end{aligned}
$$

and

$$
\begin{aligned}
\alpha_{2 i-1} & =\frac{a_{i}}{\tilde{\kappa}_{1}} e^{\tilde{\kappa}_{1} \sigma} \cosh \left(\lambda_{i} \sigma\right)+\frac{\lambda_{i}}{\tilde{\kappa}_{1}} \alpha_{2 i}, \\
\alpha_{2 i} & =\frac{a_{i}}{\tilde{\kappa}_{1}} e^{\tilde{\kappa}_{1} \sigma} \sinh \left(\lambda_{i} \sigma\right)-\frac{\lambda_{i}}{\tilde{\kappa}_{1}} \alpha_{2 i-1}
\end{aligned}
$$

for $i=2, \ldots, k$. The solutions of above the linear systems are

$$
\begin{aligned}
& \alpha_{1}=\frac{a_{1}}{\tilde{\kappa}_{1}^{2}-\lambda_{1}^{2}} e^{\tilde{\kappa}_{1} \sigma}\left(\tilde{\kappa}_{1} \cosh \left(\lambda_{1} \sigma\right)-\lambda_{1} \sinh \left(\lambda_{1} \sigma\right)\right)=\frac{a_{1}}{b_{1}} e^{\tilde{\kappa}_{1} \sigma} \sinh \theta_{1}, \\
& \alpha_{2}=\frac{a_{1}}{\tilde{\kappa}_{1}^{2}-\lambda_{1}^{2}} e^{\tilde{\kappa}_{1} \sigma}\left(\tilde{\kappa}_{1} \sinh \left(\lambda_{1} \sigma\right)-\lambda_{1} \cosh \left(\lambda_{1} \sigma\right)\right)=\frac{a_{1}}{b_{1}} e^{\tilde{\kappa}_{1} \sigma} \cosh \theta_{1},
\end{aligned}
$$

and

$$
\begin{aligned}
\alpha_{2 i-1} & =\frac{a_{i}}{\tilde{\kappa}_{1}^{2}+\lambda_{i}^{2}} e^{\tilde{\kappa}_{1} \sigma}\left(\tilde{\kappa}_{1} \cos \left(\lambda_{i} \sigma\right)+\lambda_{i} \sin \left(\lambda_{i} \sigma\right)\right)=\frac{a_{i}}{b_{i}} e^{\tilde{\kappa}_{1} \sigma} \sin \theta_{i} \\
\alpha_{2 i} & =\frac{a_{1}}{\tilde{\kappa}_{1}^{2}-\lambda_{i}^{2}} e^{\tilde{\kappa}_{1} \sigma}\left(\tilde{\kappa}_{1} \sin \left(\lambda_{i} \sigma\right)-\lambda_{i} \cos \left(\lambda_{i} \sigma\right)\right)=-\frac{a_{i}}{b_{i}} e^{\tilde{\kappa}_{1} \sigma} \cos \theta_{i} .
\end{aligned}
$$


We can get the unit vectors (26) by using the equations (11) for the timelike self-similar curve $\alpha$. Thus, we can write the following equations by means of (26)

$$
\begin{aligned}
& \mathbf{e}_{1} \cdot \mathbf{e}_{1}=-1 \quad \Rightarrow \quad-a_{1}^{2}+\sum_{i=2}^{k} a_{i}^{2}=-1, \\
& \mathbf{e}_{2} \cdot \mathbf{e}_{2}=1 \quad \Rightarrow \quad \sum_{i=1}^{k} a_{i}^{2} \lambda_{i}^{2}=1, \\
& \mathbf{e}_{3} \cdot \mathbf{e}_{3}=1 \quad \Rightarrow \quad-a_{1}^{2}\left(1-\lambda_{1}^{2}\right)^{2}+\sum_{i=2}^{k}\left(1+\lambda_{i}^{2}\right)^{2} a_{i}^{2}=\tilde{\kappa}_{2}^{2}, \\
& \mathbf{e}_{i} \cdot \mathbf{e}_{i}=1 \quad \Rightarrow \quad \text { so on }, i=4, \ldots, k .
\end{aligned}
$$

ii) The proof is similar to the proof of i).

Corollary 14. i) Let $\alpha: I \rightarrow \mathbb{E}_{1}^{2 k}(k>1)$ be a timelike self-similar curve with a parametric representation (25). Then, this curve lies on the quadratic timelike hypersurface with an equation

$$
\begin{aligned}
& \frac{b_{1}^{2}}{a_{1}^{2}}\left(-x_{1}^{2}+x_{2}^{2}\right)+\frac{b_{2}^{2}}{a_{2}^{2}}\left(x_{1}^{2}+x_{2}^{2}\right)+\cdots+\frac{b_{k-1}^{2}}{a_{k-1}^{2}}\left(x_{2 k-3}^{2}+x_{2 k-2}^{2}\right) \\
= & (k-1) \frac{b_{k}^{2}}{a_{k}^{2}}\left(x_{2 k-1}^{2}+x_{2 k}^{2}\right) .
\end{aligned}
$$

ii) Let $\alpha: I \rightarrow \mathbb{E}_{1}^{2 k}(k>1)$ be a spacelike self-similar curve with a parametric representation (27). Then, this curve lies on the quadratic Lorentzian hypersurface with an equation

$$
\begin{aligned}
& \frac{b_{1}^{2}}{a_{1}^{2}}\left(x_{1}^{2}-x_{2}^{2}\right)+\frac{b_{2}^{2}}{a_{2}^{2}}\left(x_{1}^{2}+x_{2}^{2}\right)+\cdots+\frac{b_{k-1}^{2}}{a_{k-1}^{2}}\left(x_{2 k-3}^{2}+x_{2 k-2}^{2}\right) \\
= & (k-1) \frac{b_{k}^{2}}{a_{k}^{2}}\left(x_{2 k-1}^{2}+x_{2 k}^{2}\right) .
\end{aligned}
$$

Now, we examine the following examples of the non-null self-similar curves in the Minkowski plane and Minkowski space-time.

Case $\boldsymbol{n}=\mathbf{2}$ (Lorentzian plane): Let $\alpha_{2}: I \rightarrow \mathbb{E}_{1}^{2}$ be a timelike self-similar curve with the constant invariant $\tilde{\kappa}_{1}$. Then, we can write $\lambda_{1}^{2}=1$ and $a_{1}^{2} \lambda_{1}^{2}=1$. Hence, a parametrization of $\alpha_{2}$ is

$$
\alpha_{2}(\sigma)=\left(\frac{1}{\sqrt{1-\tilde{\kappa}_{1}^{2}}} e^{\tilde{\kappa}_{1} \sigma} \sinh \theta, \frac{1}{\sqrt{1-\tilde{\kappa}_{1}^{2}}} e^{\tilde{\kappa}_{1} \sigma} \cosh \theta\right),
$$

where $\theta=\sigma+\cosh ^{-1}\left(\frac{1}{\sqrt{1-\tilde{\kappa}_{1}^{2}}}\right)$. 
Case $\boldsymbol{n}=\mathbf{4}$ (Minkowski space-time): Let $\alpha_{4}: I \rightarrow \mathbb{E}_{1}^{4}$ be a timelike self-similar curve with the constant invariants $\tilde{\kappa}_{1}, \tilde{\kappa}_{2}$ and $\tilde{\kappa}_{3}$. Then, the semisymmetric matrix

$$
M^{2}=\left[\begin{array}{cccc}
1 & 0 & \tilde{\kappa}_{2} & 0 \\
0 & 1-\tilde{\kappa}_{2}^{2} & 0 & \tilde{\kappa}_{2} \tilde{\kappa}_{3} \\
-\tilde{\kappa}_{2} & 0 & -\tilde{\kappa}_{2}^{2}-\tilde{\kappa}_{3}^{2} & 0 \\
0 & \tilde{\kappa}_{2} \tilde{\kappa}_{3} & 0 & -\tilde{\kappa}_{3}^{2}
\end{array}\right]
$$

has two eigenvalues of multiplicity 2

$$
\lambda_{i}^{2}=\frac{1}{2}\left(1-\tilde{\kappa}_{2}^{2}-\tilde{\kappa}_{3}^{2}+(-1)^{i} \sqrt{\left(1-\tilde{\kappa}_{2}^{2}-\tilde{\kappa}_{3}^{2}\right)^{2}+4 \tilde{\kappa}_{3}^{2}}\right)
$$

for $i=1,2$. The solution of system of quadratic equations

$$
\begin{aligned}
-a_{1}^{2}+a_{2}^{2} & =-1, \\
a_{1}^{2} \lambda_{1}^{2}+a_{2}^{2} \lambda_{2}^{2} & =1
\end{aligned}
$$

is given by

$$
a_{1}=\sqrt{\frac{1+\lambda_{2}^{2}}{\lambda_{1}^{2}+\lambda_{2}^{2}}}, \quad a_{2}=\sqrt{\frac{1-\lambda_{1}^{2}}{\lambda_{1}^{2}+\lambda_{2}^{2}}}
$$

since we have $1-\lambda_{1}^{2}>0$. Consequently, the spherical arc-length parametrization of the timelike self-similar curve $\alpha_{4}$ is given by

$$
\alpha_{4}(\sigma)=\left(\frac{a_{1}}{b_{1}} e^{\tilde{\kappa}_{1} \sigma} \sinh \theta, \frac{a_{1}}{b_{1}} e^{\tilde{\kappa}_{1} \sigma} \cosh \theta, \frac{a_{2}}{b_{2}} e^{\tilde{\kappa}_{1} \sigma} \sin \theta,-\frac{a_{2}}{b_{2}} e^{\tilde{\kappa}_{1} \sigma} \cos \theta\right),
$$

where $b_{1}=\sqrt{\lambda_{1}^{2}-\tilde{\kappa}_{1}^{2}}, b_{2}=\sqrt{\lambda_{2}^{2}+\tilde{\kappa}_{1}^{2}}$ and $\theta_{1}=\lambda_{1} \sigma-\cosh ^{-1}\left(\frac{\lambda_{1}}{\sqrt{\lambda_{1}^{2}-\tilde{\kappa}_{1}^{2}}}\right)$, $\theta_{2}=\lambda_{2} \sigma+\cos ^{-1}\left(\frac{\lambda_{2}}{\sqrt{\lambda_{2}^{2}+\tilde{\kappa}_{1}^{2}}}\right)$.

\subsection{Non-null self-similar curves in odd-dimensional Minkowski space}

It can be given non-null self-similar curves in $\mathbb{E}_{1}^{2 k+1}$ with the following theorem.

Theorem 15. Let $\alpha: I \rightarrow \mathbb{E}_{1}^{2 k+1}$ be a non-null self-similar curve with constant p-shape curvatures $\tilde{\kappa}_{1} \neq 0, \tilde{\kappa}_{2} \neq 0, \ldots, \tilde{\kappa}_{2 k} \neq 0$. Suppose that $\lambda_{1}^{2}, \lambda_{2}^{2}, \ldots, \lambda_{k}^{2}$ are all different eigenvalues of the symmetric matrix $M^{2}$. So,

i) If the unit vector $\mathbf{e}_{1}$ is the timelike vector, then a parametric statement of the timelike self-similar curve $\alpha$ according to the arc-length parameter $\sigma$ can be written in the form

$$
\begin{aligned}
\alpha(\sigma)=( & \frac{a_{1}}{b_{1}} e^{\tilde{\kappa}_{1} \sigma} \sinh \theta_{1}, \frac{a_{1}}{b_{1}} e^{\tilde{\kappa}_{1} \sigma} \cosh \theta_{1}, \frac{a_{2}}{b_{2}} e^{\tilde{\kappa}_{1} \sigma} \sin \theta_{2},-\frac{a_{2}}{b_{2}} e^{\tilde{\kappa}_{1} \sigma} \cos \theta_{2}, \ldots, \\
& \left.\frac{a_{k}}{b_{k}} e^{\tilde{\kappa}_{1} \sigma} \sin \theta_{k},-\frac{a_{k}}{b_{k}} e^{\tilde{\kappa}_{1} \sigma} \cos \theta_{k}, a_{k+1} e^{\tilde{\kappa}_{1} \sigma}\right),
\end{aligned}
$$


where

$$
b_{1}=\sqrt{\lambda_{1}^{2}-\tilde{\kappa}_{1}^{2}}, \quad \theta_{1}=\lambda_{1} \sigma-\cosh ^{-1}\left(\frac{\lambda_{1}}{\sqrt{\lambda_{1}^{2}-\tilde{\kappa}_{1}^{2}}}\right),
$$

and for $i=2, \ldots, k$

$$
b_{i}=\sqrt{\lambda_{i}^{2}+\tilde{\kappa}_{1}^{2}}, \quad \theta_{i}=\lambda_{i} \sigma+\cos ^{-1}\left(\frac{\lambda_{i}}{\sqrt{\lambda_{i}^{2}+\tilde{\kappa}_{1}^{2}}}\right) .
$$

The real different non-zero numbers $a_{1}, \ldots, a_{k}, a_{k+1}$ are a solution of the system of $k+1$ algebraic quadratic equations

$$
\begin{array}{ll}
\mathbf{e}_{1} \cdot \mathbf{e}_{1}=-1 & \text { or } \quad-a_{1}^{2}+\sum_{i=2}^{k} a_{i}^{2}+\tilde{\kappa}_{1}^{2} a_{k+1}^{2}=-1, \\
\mathbf{e}_{2} \cdot \mathbf{e}_{2}=1 & \text { or } \quad \sum_{i=1}^{k} a_{i}^{2} \lambda_{i}^{2}=1, \\
\mathbf{e}_{3} \cdot \mathbf{e}_{3}=1 & \text { or } \quad-a_{1}^{2}\left(1-\lambda_{1}^{2}\right)^{2}+\sum_{i=2}^{k}\left(1+\lambda_{i}^{2}\right)^{2} a_{i}^{2}+\tilde{\kappa}_{1}^{2} a_{k+1}^{2}=\tilde{\kappa}_{2}^{2}, \\
\mathbf{e}_{i} \cdot \mathbf{e}_{i}=1, & i=4, \ldots, k+1
\end{array}
$$

determined by the vectors

$$
\begin{aligned}
\mathbf{e}_{1}(\sigma) & =e^{-\tilde{\kappa}_{1} \sigma} \frac{d}{d \sigma} \alpha(\sigma), \\
\mathbf{e}_{2}(\sigma) & =\frac{d}{d \sigma} \mathbf{e}_{1}(\sigma), \\
\mathbf{e}_{3}(\sigma) & =\frac{1}{\tilde{\kappa}_{2}}\left(-\mathbf{e}_{1}(\sigma)+\frac{d}{d \sigma} \mathbf{e}_{2}(\sigma)\right), \\
\mathbf{e}_{4}(\sigma) & =\frac{1}{\tilde{\kappa}_{3}}\left(\tilde{\kappa}_{2} \mathbf{e}_{2}(\sigma)+\frac{d}{d \sigma} \mathbf{e}_{3}(\sigma)\right), \\
& \vdots \\
\mathbf{e}_{k+1}(\sigma) & =\frac{1}{\tilde{\kappa}_{k}}\left(\tilde{\kappa}_{k-1} \mathbf{e}_{k-1}(\sigma)+\frac{d}{d \sigma} \mathbf{e}_{k}(\sigma)\right) .
\end{aligned}
$$

ii) If the unit vector $\mathbf{e}_{2}$ is timelike vector, then a parametric representation of the spacelike self-similar curve $\alpha$ according to arc-length parameter $\sigma$ can be written in the form

$\alpha(\sigma)=\left(-\frac{a_{1}}{b_{1}} e^{\tilde{\kappa}_{1} \sigma} \cosh \theta_{1},-\frac{a_{1}}{b_{1}} e^{\tilde{\kappa}_{1} \sigma} \sinh \theta_{1}, \frac{a_{2}}{b_{2}} e^{\tilde{\kappa}_{1} \sigma} \sin \theta_{2},-\frac{a_{2}}{b_{2}} e^{\tilde{\kappa}_{1} \sigma} \cos \theta_{2}, \ldots\right.$,

$$
\left.\frac{a_{k}}{b_{k}} e^{\tilde{\kappa}_{1} \sigma} \sin \theta_{k},-\frac{a_{k}}{b_{k}} e^{\tilde{\kappa}_{1} \sigma} \cos \theta_{k}, a_{k+1} e^{\tilde{\kappa}_{1} \sigma}\right) .
$$


The real different non-zero numbers $a_{1}, \ldots, a_{k}, a_{k+1}$ are a solution of the system of $k+1$ algebraic quadratic equations

$$
\begin{array}{lll}
\mathbf{e}_{1} \cdot \mathbf{e}_{1}=1 & \text { or } \quad & \sum_{i=1}^{k} a_{i}^{2}+\tilde{\kappa}_{1}^{2} a_{k+1}^{2}=1, \\
\mathbf{e}_{2} \cdot \mathbf{e}_{2}=-1 & \text { or } & -a_{1}^{2}+\sum_{i=2}^{k} a_{i}^{2} \lambda_{i}^{2}=-1, \\
\mathbf{e}_{3} \cdot \mathbf{e}_{3}=1 & \text { or } \quad & a_{1}^{2}\left(1-\lambda_{1}^{2}\right)^{2}+\sum_{i=2}^{k}\left(1+\lambda_{i}^{2}\right) a_{i}^{2}+\tilde{\kappa}_{1}^{2} a_{k+1}^{2}=\tilde{\kappa}_{2}^{2}, \\
\mathbf{e}_{i} \cdot \mathbf{e}_{i}=1, & i=4, \ldots, k+1,
\end{array}
$$

determined by the vectors

$$
\begin{aligned}
\mathbf{e}_{1}(\sigma) & =e^{-\tilde{\kappa}_{1} \sigma} \frac{d}{d \sigma} \alpha(\sigma) \\
\mathbf{e}_{2}(\sigma) & =-\frac{d}{d \sigma} \mathbf{e}_{1}(\sigma) \\
\mathbf{e}_{3}(\sigma) & =\frac{1}{\tilde{\kappa}_{2}}\left(\mathbf{e}_{1}(\sigma)+\frac{d}{d \sigma} \mathbf{e}_{2}(\sigma)\right) \\
\mathbf{e}_{4}(\sigma) & =\frac{1}{\tilde{\kappa}_{3}}\left(-\tilde{\kappa}_{2} \mathbf{e}_{2}(\sigma)+\frac{d}{d \sigma} \mathbf{e}_{3}(\sigma)\right), \\
& \vdots \\
\mathbf{e}_{k}(\sigma) & =\frac{1}{\tilde{\kappa}_{k-1}}\left(\tilde{\kappa}_{k-2} \mathbf{e}_{k-2}(\sigma)+\frac{d}{d \sigma} \mathbf{e}_{k-1}(\sigma)\right) .
\end{aligned}
$$

Proof. The proof is the same as the proof of Theorem 13.

Corollary 16. i) Let $\alpha: I \rightarrow \mathbb{E}_{1}^{2 k+1}(k>1)$ be a timelike self-similar curve with a parametric representation (25). Then, this curve lies on the quadratic timelike hypersurface with an equation

$$
\begin{aligned}
& \frac{b_{1}^{2}}{a_{1}^{2}}\left(-x_{1}^{2}+x_{2}^{2}\right)+\frac{b_{2}^{2}}{a_{2}^{2}}\left(x_{1}^{2}+x_{2}^{2}\right)+\cdots+\frac{b_{k-1}^{2}}{a_{k-1}^{2}}\left(x_{2 k-3}^{2}+x_{2 k-2}^{2}\right) \\
& +\frac{b_{k}^{2}}{a_{k}^{2}}\left(x_{2 k-1}^{2}+x_{2 k}^{2}\right)=\frac{k}{a_{k+1}^{2}} x_{2 k+1}^{2} .
\end{aligned}
$$

ii) Let $\alpha: I \rightarrow \mathbb{E}_{1}^{2 k}(k>1)$ be a spacelike self-similar curve with a parametric representation (27). Then, this curve lies on the quadratic Lorentzian hypersurface with an equation

$$
\frac{b_{1}^{2}}{a_{1}^{2}}\left(x_{1}^{2}-x_{2}^{2}\right)+\frac{b_{2}^{2}}{a_{2}^{2}}\left(x_{1}^{2}+x_{2}^{2}\right)+\cdots+\frac{b_{k-1}^{2}}{a_{k-1}^{2}}\left(x_{2 k-3}^{2}+x_{2 k-2}^{2}\right)
$$




$$
+\frac{b_{k}^{2}}{a_{k}^{2}}\left(x_{2 k-1}^{2}+x_{2 k}^{2}\right)=\frac{k}{a_{k+1}^{2}} x_{2 k+1}^{2} .
$$

Now, we investigate the timelike self-similar curves in the Minkowski 3-space $\mathbb{E}_{1}^{3}$.

Case $\boldsymbol{n}=\mathbf{3}$ (Minkowski 3-space): Let $\alpha_{3}: I \rightarrow \mathbb{E}_{1}^{3}$ be a timelike self-similar curve with constant invariant $\tilde{\kappa}_{1} \neq 0$ and $\tilde{\kappa}_{2} \neq 0$. Then, the semi-symmetric matrix

$$
M^{2}=\left[\begin{array}{ccc}
1 & 0 & \tilde{\kappa}_{2} \\
0 & 1-\tilde{\kappa}_{2}^{2} & 0 \\
-\tilde{\kappa}_{2} & 0 & -\tilde{\kappa}_{2}^{2}
\end{array}\right]
$$

has a unique non-zero eigenvalue of multiplicity 2

$$
\lambda_{1}^{2}=1-\tilde{\kappa}_{2}^{2}
$$

and therefore $b_{1}=\sqrt{1-\tilde{\kappa}_{1}^{2}-\tilde{\kappa}_{2}^{2}}$. By the Theorem 15 , we can compute

$$
a_{1}=\sqrt{\frac{1}{1-\tilde{\kappa}_{2}^{2}}}, \quad a_{2}=\sqrt{\frac{\tilde{\kappa}_{2}^{2}}{\tilde{\kappa}_{1}^{2}\left(1-\tilde{\kappa}_{2}^{2}\right)}} .
$$

Hence, a parametrization of $\alpha_{3}$ with respect to spherical arc-lentgh is given by $(32)$

$\alpha_{3}(\sigma)=\left(\frac{e^{\tilde{\kappa}_{1} \sigma} \sinh \theta}{\sqrt{\left(1-\tilde{\kappa}_{2}^{2}\right)\left(1-\tilde{\kappa}_{1}^{2}-\tilde{\kappa}_{2}^{2}\right)}}, \frac{e^{\tilde{\kappa}_{1} \sigma} \cosh \theta}{\sqrt{\left(1-\tilde{\kappa}_{2}^{2}\right)\left(1-\tilde{\kappa}_{1}^{2}-\tilde{\kappa}_{2}^{2}\right)}}, \sqrt{\frac{\tilde{\kappa}_{2}^{2}}{\tilde{\kappa}_{1}^{2}\left(1-\tilde{\kappa}_{2}^{2}\right)}} e^{\tilde{\kappa}_{1} \sigma}\right)$

where $\theta=\sigma \sqrt{1-\tilde{\kappa}_{2}^{2}}+\cosh ^{-1} \sqrt{\frac{1-\tilde{\kappa}_{1}^{2}}{1-\tilde{\kappa}_{1}^{2}-\tilde{\kappa}_{2}^{2}}}$. It is clear that the timelike selfsimilar curve $\alpha_{3}$ is a curve on the surface with an implicit equation

$$
\frac{\tilde{\kappa}_{2}^{2}}{\tilde{\kappa}_{1}^{2}}\left(-x_{1}^{2}+x_{2}^{2}\right)=\frac{1}{1-\tilde{\kappa}_{1}^{2}-\tilde{\kappa}_{2}^{2}} x_{3}^{2}
$$

(see Figure 2).

\section{Concluding remarks}

In this paper, we gave the p-similarity invariants of a non-null curves in the Lorentzian n-space. We also proved the fundamental existence and uniqueness theorems for non-null curves under p-similarity transformation. We studied self-similar non-null curves in Lorentzian n-space. We think that the notion of similarity and self-similarity in the Lorentzian-Minkowski space may form many new ideas and concepts to the pure and applied mathematics.

Bejancu [5] represented a method for the general study of the geometry of null curves in Lorentz manifolds and, more generally, in semi-Riemannian manifolds (see also [11]). A. Ferrandez, A. Gimenez, and P. Lucas [15] generalized the Cartan frame to Lorentzian space forms. They showed the fundamental existence and uniqueness theorems and they obtained values of the Cartan curvatures in higher dimensions. Therefore, it is of interest to investigate the 


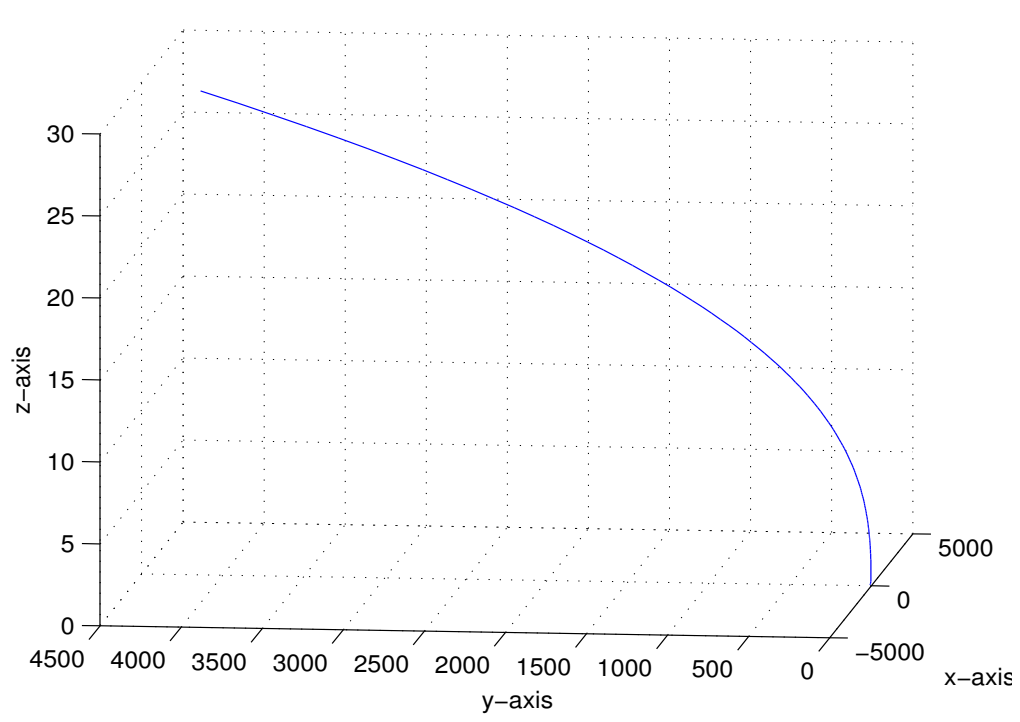

Figure 2. The timelike self-similar curve parameterized by (32).

similarity invariants of null curves in Lorentzian n-space under these considerations.

The motions of curves in $\mathbb{E}^{2}, \mathbb{E}^{3}$ and $\mathbb{E}^{n}(n>3)$ yield the mKdV hierarchy, Schrödinger hierarchy and a multi- component generalization of mKdVSchrödinger hierarchies, respectively. K.-S. Chou and C. Qu [9] showed that the motions of curves in two-, three- and n-dimensional $(n>3)$ similarity geometries correspond to the Burgers hierarchy, Burgers-mKdV hierarchy and a multi-component generalization of these hierarchies by using the similarity invariants of curves in comparison with its invariants under the Euclidean motion. Also, they [8] found that many 1+1-dimensional integrable equations like $\mathrm{KdV}$, Burgers, Sawada-Kotera, Harry-Dym hierarchies and Camassa-Holm equations arise from motions of plane curves in centro-affine, similarity, affine and fully affine geometries. The motion of curves on two-dimensional surfaces in $\mathbb{E}_{1}^{3}$ was considered by Gürses [18]. Q. Ding and J. Inoguchi [10] showed binormal motions of curves in Minkowski 3-space are closely related to Schrödinger flows into the Lorentzian symmetric space and Riemannian symmetric space. Therefore, with the aid of the current paper, it will be studied the motion of Lorentzian similar curves with p-similarity invariants under the consideration of the paper [9].

\section{References}

[1] J. G. Alcázar, C. Hermosoa, and G. Muntinghb, Detecting similarity of rational plane curves, J. Comput. Appl. Math. 269 (2014), 1-13. 
[2] T. Aristide, Closed similarity Lorentzian Affine manifolds, Proc. Amer. Math. Soc. 132 (2004), no. 12, 3697-3702.

[3] M. F. Barnsley and S. Demko, Iterated function systems and the global construction of fractals, Proc. Roy. Soc. London Ser. A 399 (1985), no. 1817, 243-275.

[4] M. F. Barnsley, J. E. Hutchinson, and Ö. Stenflo, V-variable fractals: Fractals with partial self similarity, Adv. Math. 218 (2008), no. 6, 2051-2088.

[5] A. Bejancu, Lightlike curves in Lorentz manifolds, Publ. Math. Debrecen 44 (1994), no. 1-2, 145-155.

[6] M. Berger, Geometry I, Springer, New York 1987.

[7] A. Brook, A. M. Bruckstein, and R. Kimmel, On similarity-invariant fairness measures, LNCS 3459, pp. 456-467, 2005.

[8] K.-S. Chou and C. Qu, Integrable equations arising from motions of plane curves, Phys. D 162 (2002), no. 1-2, 9-33.

[9] _ Motions of curves in similarity geometries and Burgers-mKdV hierarchies, Chaos Solitons Fractals 19 (2004), no. 1, 47-53.

[10] Q. Ding and J. Inoguchi, Schrödinger flows, binormal motion for curves and the second AKNS-hierarchies, Chaos Solitons Fractals 21 (2004), no. 3, 669-677.

[11] K. L. Duggal and A. Bejancu, Lightlike Submanifolds of Semi-Riemannian Manifolds and Applications, Volume 364 of Mathematics and its Aplications. Kluwer Academic Publishers Group, Dordrecht, The Netherlands, 1996.

[12] R. Encheva and G. Georgiev, Shapes of space curves, J. Geom. Graph. 7 (2003), no. 2, $145-155$.

[13] Similar Frenet curves, Results Math. 55 (2009), no. 3-4, 359-372.

[14] K. Falconer, Fractal Geometry, Second Edition, John Wiley \& Sons, Ltd., 2003.

[15] A. Ferrandez, A. Gimenez, and P. Lucas, Null helices in Lorentzian space forms, Int. J. Mod. Phys. A 16 (2001), 4845-4863.

[16] W. Greub, Linear Algebra, 3rd ed., Springer Verlag, Heidelberg, 1967.

[17] R. Grigorchuk and Z. Sunic, Self Similarity an branching group theory, Volume 1, London Mathematical Society Lecture Note Series: 339, Groups St Andrews 2005.

[18] M. Gürses, Motion of curves on two-dimensional surfaces and soliton equations, Phys. Lett. A 241 (1998), no. 6, 329-334.

[19] J. E. Hutchinson, Fractals and self-similarity, Indiana Univ. Math. J. 30 (1981), no. 5, $713-747$.

[20] Y. Kamishima, Lorentzian similarity manifolds, Cent. Eur. J. Math. 10 (2012), no. 5, 1771-1788.

[21] S. Z. Li, Similarity invariants for 3D space curve matching, In Proceedings of the First Asian Conference on Computer Vision, pp. 454-457, Japan 1993.

[22] _ Invariant representation, matching and pose estimation of $3 D$ space curves under similarity transformation, Pattern Recognition 30 (1997), no. 3, 447-458.

[23] B. B. Mandelbrot, The Fractal Geometry of Nature, New York: W. H. Freeman, 1982.

[24] K. Nakayama, Motion of curves in hyperboloid in the Minkowski space, J. Phys. Soc. Japan 67 (1998), no. 9, 3031-3037.

[25] V. Nekrashevych, Self-similar groups and their geometry, São Paulo J. Math. Sci. 1 (2007), no. 1, 41-95.

[26] B. O'Neill, Semi-Riemannian Geometry, Academic Press Inc., London, 1983.

[27] M. Özdemir, On the focal curvatures of non-lightlike curves in Minkowski $(m+1)$-space, F. Ü. Fen ve Mühendislik Bilimleri Dergisi 16 (2004), no. 3, 401-409.

[28] H. Sahbi, Kernel PCA for similarity invariant shape recognition, Neurocomputing 70 (2007), 3034-3045.

[29] D. A. Singer and D. H. Steinberg, Normal forms in Lorentzian spaces, Nova J. Algebra Geom. 3 (1994), no. 1, 1-9. 
[30] D. Xu and $\mathrm{H}$. Li, 3-D curve moment invariants for curve recognition, Lecture Notes in Control and Information Sciences, 345, pp. 572-577, 2006.

Mustafa Özdemir

Department of Mathematics

AKDENIZ UNIVERSity

Antalya, Turkey

E-mail address: mozdemir@akdeniz.edu.tr

HAKAN SimseK

Department of Mathematics

Akdeniz University

Antalya, Turkey

E-mail address: hakansimsek@akdeniz.edu.tr 